PREPARED FOR THE U.S. DEPARTMENT OF ENERGY, UNDER CONTRACT DE-AC02-76CH03073

PPPL-3790

PPPL-3790

UC-70

Truncated Thermal Equilibrium Distribution for Intense Beam Propagation

by

R.C. Davidson, H. Qin, and Steven M. Lund

February 2003

NM|

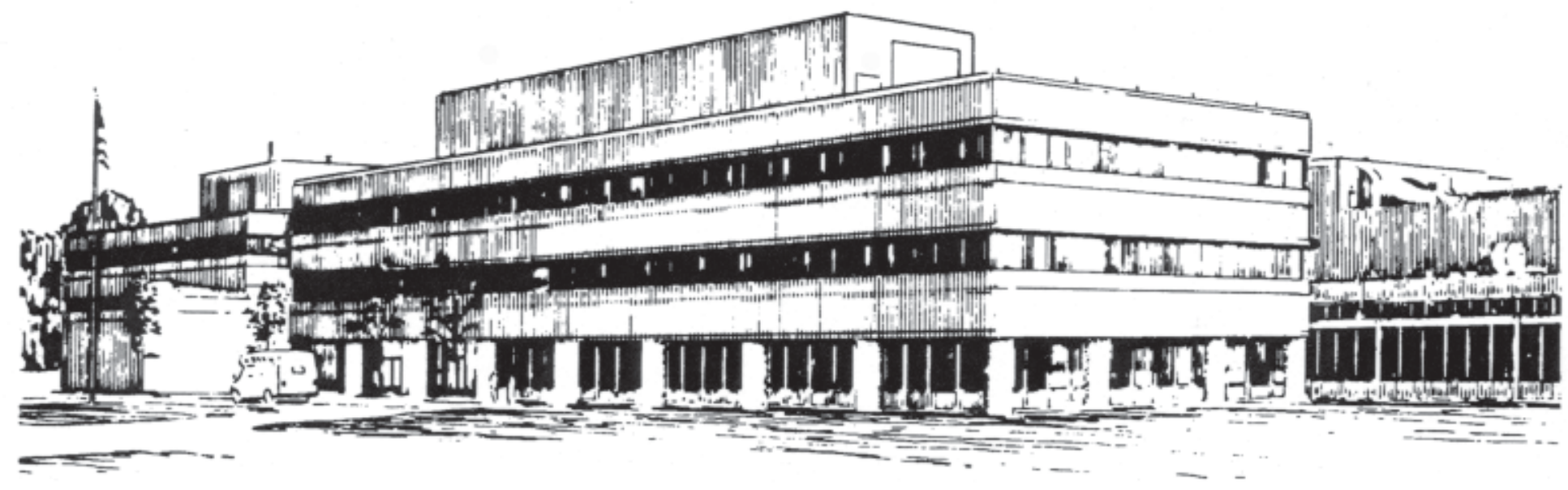

PRINCETON PLASMA PHYSICS LABORATORY PRINCETON UNIVERSITY, PRINCETON, NEW JERSEY 


\section{PPPL Reports Disclaimer}

This report was prepared as an account of work sponsored by an agency of the United States Government. Neither the United States Government nor any agency thereof, nor any of their employees, makes any warranty, express or implied, or assumes any legal liability or responsibility for the accuracy, completeness, or usefulness of any information, apparatus, product, or process disclosed, or represents that its use would not infringe privately owned rights. Reference herein to any specific commercial product, process, or service by trade name, trademark, manufacturer, or otherwise, does not necessarily constitute or imply its endorsement, recommendation, or favoring by the United States Government or any agency thereof. The views and opinions of authors expressed herein do not necessarily state or reflect those of the United States Government or any agency thereof.

\section{Availability}

This report is posted on the U.S. Department of Energy's Princeton Plasma Physics Laboratory Publications and Reports web site in Fiscal Year 2003. The home page for PPPL Reports and Publications is: http://www.pppl.gov/pub_report/

DOE and DOE Contractors can obtain copies of this report from:

U.S. Department of Energy

Office of Scientific and Technical Information

DOE Technical Information Services (DTIS)

P.O. Box 62

Oak Ridge, TN 37831

Telephone: (865) 576-8401

Fax: (865) 576-5728

Email: reports@adonis.osti.gov

This report is available to the general public from:

National Technical Information Service

U.S. Department of Commerce

5285 Port Royal Road

Springfield, VA 22161

Telephone: $1-800-553-6847$ or

(703) $605-6000$

Fax: (703) 321-8547

Internet: http://www.ntis.gov/ordering.htm 


\title{
Truncated Thermal Equilibrium Distribution for Intense Beam Propagation
}

\author{
Ronald C. Davidson and Hong Qin \\ Plasma Physics Laboratory, Princeton University, Princeton, NJ 08543 \\ Steven M. Lund \\ Lawrence Livermore National Laboratory, \\ University of California, Livermore, CA 94550
}

\begin{abstract}
An intense charged particle beam with directed kinetic energy $\left(\gamma_{b}-1\right) m_{b} c^{2}$ propagates in the $z$-direction through an applied focusing field with transverse focusing force modeled by $\mathbf{F}_{f o c}=$ $-\gamma_{b} m_{b} \omega_{\beta \perp}^{2} \mathbf{x}_{\perp}$ in the smooth focusing approximation. This paper examines properties of the axisymmetric, truncated thermal equilibrium distribution $F_{b}\left(r, \mathbf{p}_{\perp}\right)=A \exp \left(-H_{\perp} / \hat{T}_{\perp b}\right) \oplus\left(H_{\perp}-E_{b}\right)$, where $A, \hat{T}_{\perp b}$ and $E_{b}$ are positive constants, and $H_{\perp}$ is the Hamiltonian for transverse particle motion. The equilibrium profiles for beam number density, $n_{b}(r)=\int d^{2} p F_{b}\left(r, \mathbf{p}_{\perp}\right)$, and transverse temperature, $T_{\perp b}(r)=\int d^{2} p\left(p_{\perp}^{2} / 2 \gamma_{b} m_{b}\right) F_{b}\left(r, \mathbf{p}_{\perp}\right)$, are calculated self-consistently including spacecharge effects. Several properties of the equilibrium profiles are noteworthy. For example, the beam has a sharp outer edge radius $r_{b}$ with $n_{b}\left(r \geq r_{b}\right)=0$, where $r_{b}$ depends on the value of $E_{b} / \hat{T}_{\perp b}$. In addition, unlike the choice of a semi-Gaussian distribution, $F_{b}^{S G}=A \exp \left(-p_{\perp}^{2} / 2 \gamma_{b} m_{b} \hat{T}_{\perp b}\right) \oplus\left(r-r_{b}\right)$, the truncated thermal equilibrium distribution $F_{b}(r, \mathbf{p})$ depends on $(r, \mathbf{p})$ only through the singleparticle constant of the motion $H_{\perp}$ and is therefore a true steady-state solution $(\partial / \partial t=0)$ of the nonlinear Vlasov-Maxwell equations.
\end{abstract}




\section{INTRODUCTION AND THEORETICAL MODEL}

This paper makes use of the nonlinear Vlasov-Maxwell equations[1-4] to investigate the properties of self-consistent intense beam equilibria $(\partial / \partial t=0)$ with sharp outer beam edge at radius $r=r_{b}$. The analysis considers an axially continuous charged particle beam made up of particles with charge $e_{b}$ and rest mass $m_{b}$ propagating in the $z$-direction with directed axial kinetic energy $\left(\gamma_{b}-1\right) m_{b} c^{2}$, where $\gamma_{b}=\left(1-\beta_{b}^{2}\right)^{-1 / 2}$ is the relativistic mass factor, $V_{b}=\beta_{b} c$ is the average axial velocity of the beam particles, and $c$ is the speed of light in vacuo. The applied transverse focusing force on a beam particle is modeled in the smooth-focusing approximation[1-3] by $\mathbf{F}_{f o c}=-\gamma_{b} m_{b} \omega_{\beta \perp}^{2} \mathbf{x}_{\perp}$, where $\omega_{\beta \perp}=$ const. is the applied focusing frequency, and $\mathbf{x}_{\perp}=x \hat{\mathbf{e}}_{x}+y \hat{\mathbf{e}}_{y}$ is the transverse displacement of a beam particle from the axis. Finally, the particle motion in the beam frame is assumed to be nonrelativistic. The main purpose of the present analysis is to describe a particular choice of self-consistent beam equilibrium distribution function, with sharp outer beam edge, that can serve as a useful alternative to a semi-Gaussian distribution.

By way of background, particle-in-cell simulations of intense beam propagation[5-9] often make use of an input distribution function, known as a semi-Gaussian distribution, in which the phase-space dependence of the transverse distribution function is taken to be of the form

$$
F_{b}^{S G}=\frac{\hat{n}_{b}}{2 \pi \gamma_{b} m_{b} \hat{T}_{\perp b}} \exp \left(-\frac{p_{\perp}^{2}}{2 \gamma_{b} m_{b} \hat{T}_{\perp b}}\right) \oplus\left(r-r_{b}\right) .
$$

Here, $p_{\perp}=\left(p_{x}^{2}+p_{y}^{2}\right)^{1 / 2}$ is the transverse particle momentum, $r=\left(x^{2}+y^{2}\right)^{1 / 2}$ is the radial distance of a particle from the beam axis, $\hat{n}_{b}$ and $\hat{T}_{\perp b}$ are positive constants, and $\oplus(x)$ is the Heaviside step-function defined by $\oplus(x)=+1$ for $x<0$, and $\oplus(x)=0$ for $x>0$. An important feature of Eq. (1) is that the corresponding number density of beam particles, $n_{b}(r)=\int d^{2} p_{\perp} F_{b}^{S G}$, has the simple uniform-density step-function profile, $n_{b}(r)=\hat{n}_{b} \oplus(r-$ $\left.r_{b}\right)$, with a sharp beam edge at radius $r=r_{b}$, and $n_{b}\left(r>r_{b}\right)=0$. Another feature of Eq. (1) is that the local transverse temperature within the beam is spatially uniform with $\int d^{2} p\left(p_{\perp}^{2} / 2 \gamma_{b} m_{b}\right) F_{b}^{S G}=\hat{T}_{\perp b} n_{b}(r)$, where $\hat{T}_{\perp b}=$ const. A disadvantage of Eq. (1), of course, is that an input distribution function such as Eq. (1) does not correspond to a quasi-steadystate equilibrium $(\partial / \partial t=0)$, since $F_{b}^{S G}$ is not constructed from single-particle constants of the motion $[1,2,10]$. Because $\partial F_{b}^{S G} / \partial t \neq 0$, particle-in-cell simulations based on the initial

distribution $F_{b}^{S G}$ can have a significant transient evolution of the distribution function, even 
in circumstances where the beam propagation is nominally stable [9].

Consistent with the assumptions summarized above, a self-consistent equilibrium solution $(\partial / \partial t=0)$ to the nonlinear Vlasov-Maxwell equations can be constructed from the single-particle constants of the motion in the equilibrium field configuration. For an axisymmetric beam $(\partial / \partial \theta=0)$, the constants of the motion involving the transverse particle dynamics correspond to the canonical angular momentum $P_{\theta}=x p_{y}-y p_{x}$, and the transverse Hamiltonian $H_{\perp}$ defined by

$$
H_{\perp}=\frac{p_{\perp}^{2}}{2 \gamma_{b} m_{b}}+\psi(r)
$$

where

$$
\psi(r)=\frac{1}{2} \gamma_{b} m_{b} \omega_{\beta \perp}^{2} r^{2}+\frac{e_{b}}{\gamma_{b}^{2}} \phi(r)
$$

is the effective radial potential. In Eq. (2), the equilibrium space-charge potential $\phi(r)$ is determined self-consistently in terms of the equilibrium distribution function $F_{b}\left(r, \mathbf{p}_{\perp}\right)$ from Poisson's equation

$$
\frac{1}{r} \frac{\partial}{\partial r} r \frac{\partial}{\partial r} \phi(r)=-e_{b} \int d^{2} p_{\perp} F_{b}\left(r, \mathbf{p}_{\perp}\right)
$$

where $n_{b}(r)=\int d^{2} p_{\perp} F_{b}\left(r, \mathbf{p}_{\perp}\right)$ is the number density of beam particles. In solving Eq. (3), we take $\phi(r=0)=0=\psi(r=0)$ without loss of generality. Any transverse distribution function $F_{b}\left(r, \mathbf{p}_{\perp}\right)$ that depends on $\left(r, \mathbf{p}_{\perp}\right)$ only through the variables $H_{\perp}$ and $P_{\theta}$ is an exact solution to the steady-state nonlinear Vlasov-Maxwell equations[1, 2, 10]. For a non-rotating beam, this dependence occurs only through the perpendicular Hamiltonian $H_{\perp}$, i.e.,

$$
F_{b}\left(r, \mathbf{p}_{\perp}\right)=F_{b}\left(H_{\perp}\right)
$$

which is the class of beam equilibria considered in the present analysis.

The organization of this paper is the following. In Sec. 2, the truncated thermal equilibrium distribution is examined analytically. The implications of global radial force balance is considered in Sec. 3. Finally, in Sec. 4, numerical solutions are obtained for the self-field potential and radial density profile over a wide range of system parameters.

\section{TRUNCATED THERMAL EQUILIBRIUM DISTRIBUTION}

Equations (2)-(4) have been extensively analyzed in the literature[11-19] for the case of a thermal equilibrium distribution, $F_{b}\left(H_{\perp}\right)=A^{\prime} \exp \left(-H_{\perp} / \hat{T}_{\perp b}\right)$, and for a waterbag equilibrium distribution, $F_{b}\left(H_{\perp}\right)=A^{\prime \prime} \oplus\left(H_{\perp}-E_{b}\right)$, where $A^{\prime}, A^{\prime \prime}, \hat{T}_{\perp b}$ and $E_{b}$ are positive constants. 
One characteristic of the thermal equilibrium distribution is that the (bell-shaped) density profile has an exponentially small (but non-zero) value beyond the beam edge. While the waterbag equilibrium has a sharp beam edge at radius $r=r_{b}$, there is no variation of $F_{b}\left(H_{\perp}\right)$ with energy $H_{\perp}$ in the beam interior. In the present paper, we combine these two distribution functions into a single so-called truncated thermal equilibrium distribution $F_{b}\left(H_{\perp}\right)$ defined by

$$
F_{b}\left(H_{\perp}\right)=\frac{\hat{n}_{b}}{\left(2 \pi \gamma_{b} m_{b} \hat{T}_{\perp b}\right)\left[1-\exp \left(-E_{b} / \hat{T}_{\perp b}\right)\right]} \exp \left(-H_{\perp} / \hat{T}_{\perp b}\right) \oplus\left(H_{\perp}-E_{b}\right) .
$$

Here, $\hat{n}_{b}, \hat{T}_{\perp b}$, and $E_{b}$ positive constants, and $\hat{n}_{b} \equiv n_{b}(r=0)$ can be identified with the onaxis $(r=0)$ value of number density because $\phi(r=0)=0=\psi(r=0)$ is assumed. Equation $(5)$ is a fully self-consistent equilibrium solution $(\partial / \partial t=0)$ to the nonlinear Vlasov-Maxwell equations within the context of the assumptions enumerated earlier.

Substituting Eq. (5) into the definition of $n_{b}(r)=2 \pi \int_{0}^{\infty} d p_{\perp} p_{\perp} F_{b}\left(H_{\perp}\right)$ and carrying out the integration over $p_{\perp}$ readily gives the equilibrium density profile

$$
n_{b}(r)= \begin{cases}\hat{n}_{b} \frac{\left[\exp \left(-\psi / \hat{T}_{\perp b}\right)-\exp \left(-E_{b} / \hat{T}_{\perp b}\right)\right]}{\left[1-\exp \left(-E_{b} / \hat{T}_{\perp b}\right)\right]}, & 0 \leq r<r_{b}, \\ 0, & r_{b}<r \leq r_{w},\end{cases}
$$

where $r_{w}=$ const. is the radius of a cylindrical, perfectly conducting wall. Here, the outer edge radius of the beam $\left(r=r_{b}\right)$ is determined self-consistently in terms of the constant $E_{b}$ from

$$
\psi\left(r=r_{b}\right)=E_{b} \equiv \psi_{b}=\text { const } .
$$

This follows for the choice of distribution function in Eq. (5) because the particle motion is constrained to $H_{\perp}<E_{b}$, or equivalently, $p_{\perp}^{2} / 2 \gamma_{b} m_{b}<E_{b}-\psi(r)$. Therefore, the edge radius $r_{b}$ is determined from $\psi\left(r=r_{b}\right)=E_{b}$ (where $p_{\perp}=0$ ), and there are no particles for $r>r_{b}$. We substitute Eqs. (2) and (6) into Poisson's equation (3). This readily gives the closed nonlinear differential equation for $\psi(r)$,

$$
\frac{1}{r} \frac{\partial}{\partial r} r \frac{\partial}{\partial r} \frac{\psi(r)}{\hat{T}_{\perp b}}=\frac{2 \gamma_{b} m_{b} \omega_{\beta \perp}^{2}}{\hat{T}_{\perp b}}\left[1-\hat{s}_{b} \frac{\left[\exp \left(-\psi / \hat{T}_{\perp b}\right)-\exp \left(-E_{b} / \hat{T}_{\perp b}\right)\right]}{\left[1-\exp \left(-E_{b} / \hat{T}_{\perp b}\right)\right]} \oplus\left(r-r_{b}\right)\right] .
$$

In Eq. (8), the constant

$$
\hat{s}_{b}=\frac{\hat{\omega}_{p b}^{2}}{2 \gamma_{b}^{2} \omega_{\beta \perp}^{2}}
$$


is a dimensionless measure of the normalized beam intensity, and $\hat{\omega}_{p b}=\left(4 \pi \hat{n}_{b} e_{b}^{2} / \gamma_{b} m_{b}\right)^{1 / 2}$ is the relativistic plasma frequency at $r=0$. A careful examination of Eqs. (6) and (8) shows that the condition for existence of a radially confined density profile [the condition for $n_{b}(r)$ to be a non-increasing function of $r$ ] is that the normalized beam intensity satisfy $\hat{s}_{b}<1$. From Eq. (8), the inequality $\hat{s}_{b}<1$ assures that $\left\{r^{-1}(\partial / \partial r)[r \partial \psi / \partial r]\right\}_{r=0}>0$ and hence that $n_{b}(r)$ decreases monotonically as a function of increasing $r$ (see also discussion on pages 200 - 201 of Ref. 1). Whenever the inequality $\hat{s}_{b}<1$ is satisfied, the solution to Eq. (8) for $\psi(r)$ increases monotonically from $\psi(r=0)=0$ to the value $\psi\left(r=r_{b}\right)=E_{b} \equiv \psi_{b}$ at the outer edge of the beam. Correspondingly, from Eq. (6), the number density $n_{b}(r)$ decreases from the value $n_{b}(r=0)=\hat{n}_{b}$ at $r=0$, to $n_{b}\left(r=r_{b}\right)=0$ at the beam edge.

Other equilibrium properties can also be calculated for the choice of truncated thermal equilibrium distribution in Eq. (5). For example, the transverse pressure profile is defined by $P_{\perp b}(r)=n_{b}(r) T_{\perp b}(r)=2 \pi \int_{0}^{\infty} d p_{\perp} p_{\perp}\left(p_{\perp}^{2} / 2 \gamma_{b} m_{b}\right) F_{b}\left(H_{\perp}\right)[10,18]$. Making use of Eqs. (5) and (6), and integrating over $p_{\perp}$, some straightforward algebraic manipulation shows that the transverse temperature profile $T_{\perp b}(r)$ for the choice of distribution function in Eq. (5) is given by

$$
T_{\perp b}(r)=\hat{T}_{\perp b} \frac{\left\{\exp \left(-\psi / \hat{T}_{\perp b}\right)-\left[1+\left(E_{b}-\psi\right) / \hat{T}_{\perp b}\right] \exp \left(-E_{b} / \hat{T}_{\perp b}\right)\right\}}{\left[\exp \left(-\psi / \hat{T}_{\perp b}\right)-\exp \left(-E_{b} / \hat{T}_{\perp b}\right)\right]}
$$

for $0 \leq r<r_{b}$. From $\psi(r=0)=0$, we note from Eq. (10) that the on-axis value of transverse temperature is $T_{\perp b}(r=0)=\hat{T}_{\perp b}\left\{1-\left(E_{b} / \hat{T}_{\perp b}\right) /\left[\exp \left(E_{b} / \hat{T}_{\perp b}\right)-1\right]\right\}$. Furthermore, for finite value of $E_{b} / \hat{T}_{\perp b}$, it can be shown directly from Eq. (10) that the transverse temperature near the beam edge decreases monotonically to zero value, $T_{\perp b}\left(r=r_{b}\right)=0$, with $T_{\perp b}(r) \simeq$ $(1 / 2)\left[E_{b}-\psi(r)\right]$ as $r \rightarrow r_{b}$ and $\psi(r) \rightarrow E_{b}=\psi\left(r_{b}\right)$.

For specified values of the dimensionless parameters $\hat{s}_{b}=\hat{\omega}_{p b}^{2} / 2 \gamma_{b}^{2} \omega_{\beta \perp}^{2}$ and $E_{b} / \hat{T}_{\perp b}$, Eq. (8) can be solved numerically for $\psi(r) / \hat{T}_{\perp b}$, and the corresponding density profile $n_{b}(r)$ and transverse temperature profile $T_{\perp b}(r)$ determined self-consistently from Eqs. (6) and (10), respectively, over a wide range of system parameters. Of course, in the limiting case $E_{b} / \hat{T}_{\perp b} \rightarrow \infty$, Eqs. (6) and (10) reduce to the bell-shaped density profile, $n_{b}(r)=\hat{n}_{b} \exp \left[-\psi(r) / \hat{T}_{\perp b}\right]$, and uniform transverse temperature profile, $T_{\perp b}(r)=\hat{T}_{\perp b}=$ const., corresponding to thermal equilibrium[10, 18]. On the other hand, as noted earlier, for finite value of $E_{b} / \hat{T}_{\perp b}$ the beam equilibrium described by Eqs. (5), (6), (8) and (10) has a sharp radial edge at $r=r_{b}$ determined from $\psi\left(r=r_{b}\right)=E_{b}$. Moreover, the density and 
temperature profiles in Eqs. (6) and (10) decrease monotonically to zero as $r \rightarrow r_{b}$. This is expected for the choice of distribution function in Eq. (5) because the maximum transverse Hamiltonian is $\left[H_{\perp}\right]_{\max }=E_{b}$, and the radial location $r=r_{b}$ corresponds to the envelope of turning points in the transverse particle orbits for which $\left[p_{\perp}^{2}\right]=0$ and $\psi\left(r=r_{b}\right)=E_{b}$ [see Eq. (2)].

\section{GLOBAL RADIAL FORCE BALANCE}

Before examining specific numerical solutions to Eqs. (6), (8) and (10), we summarize here an important constraint corresponding to global radial force balance[20, 21] satisfied by the entire class of beam equilibria described by Eq. (4). We define the statistical average of a phase function $\chi$ by

$$
\langle\chi\rangle=\frac{1}{N_{b}} \int d^{2} x d^{2} p \chi F_{b}\left(H_{\perp}\right),
$$

where $N_{b}=\int d^{2} x d^{2} p F_{b}\left(H_{\perp}\right)=2 \pi \int_{0}^{r_{b}} d r r n_{b}(r)$ is the number of beam particles per unit axial length, and $n_{b}(r)=2 \pi \int_{0}^{\infty} d p_{\perp} p_{\perp} F_{b}\left(H_{\perp}\right)$ is the number density. Therefore, from Eq. (11), the mean-square beam radius $R_{b}^{2}=\left\langle r^{2}\right\rangle$ and the average transverse kinetic energy $\left\langle\left(p_{x}^{2}+p_{y}^{2}\right) / 2 \gamma_{b} m_{b}\right\rangle$ can be expressed as

$$
\begin{gathered}
R_{b}^{2}=\left\langle r^{2}\right\rangle=\frac{2 \pi}{N_{b}} \int_{0}^{r_{b}} d r r r^{2} n_{b}(r), \\
\left\langle\frac{p_{x}^{2}+p_{y}^{2}}{2 \gamma_{b} m_{b}}\right\rangle=\left\langle T_{\perp b}(r)\right\rangle=\frac{2 \pi}{N_{b}} \int_{0}^{r_{b}} d r r T_{\perp b}(r) n_{b}(r),
\end{gathered}
$$

where $n_{b}(r) T_{\perp b}(r)=P_{\perp b}(r)=2 \pi \int_{0}^{\infty} d p_{\perp} p_{\perp}\left(p_{\perp}^{2} / 2 \gamma_{b} m_{b}\right) F_{b}\left(H_{\perp}\right)$ is the transverse pressure profile. For the general class of equilibrium distributions in Eq. (4), it can be shown that local radial force balance on beam fluid element is given by[10, 20, 21] $\partial P_{\perp b} / \partial r=-\gamma_{b} m_{b} \omega_{\beta \perp}^{2} n_{b} r-$ $\left(n_{b} e_{b} / \gamma_{b}^{2}\right) \partial \phi / \partial r$. Without presenting algebraic details, operating with $\left(2 \pi / N_{b}\right) \int_{0}^{r_{b}} d r r \cdots$ gives the exact global radial force balance condition[10, 20, 21]

$$
\gamma_{b} m_{b} \omega_{\beta \perp}^{2} R_{b}^{2}=\frac{N_{b} e_{b}^{2}}{\gamma_{b}^{2}}+2\left\langle T_{\perp b}(r)\right\rangle,
$$

where $R_{b}^{2}$ and $\left\langle T_{\perp b}(r)\right\rangle$ are defined in Eq. (12).

Equation (13) shows clearly that there are two contributions to the mean-square beam radius $R_{b}^{2}=\left\langle r^{2}\right\rangle$. The term proportional to $N_{b} e_{b}^{2} / \gamma_{b}^{2}=N_{b} e_{b}^{2}\left(1-\beta_{b}^{2}\right)$ corresponds to the spacecharge contribution $\left(N_{b} e_{b}^{2}\right)$ reduced by self-magnetic field effects $\left(-N_{b} e_{b}^{2} \beta_{b}^{2}\right)$, and the term 
proportional to $2\left\langle T_{\perp b}(r)\right\rangle$ corresponds to the emittance contribution, proportional to the average transverse temperature of the beam particles. Indeed, it is instructive to introduce the familiar quantities corresponding to self-field perveance $\left(K_{b}\right)$, smooth-focusing lattice coefficient $\left(\kappa_{s f}\right)$, and unnormalized beam emittance $(\epsilon)$ defined by

$$
\begin{aligned}
K_{b} & \equiv \frac{2 N_{b} e_{b}^{2}}{\gamma_{b}^{3} m_{b} \beta_{b}^{2} c^{2}}, \quad \kappa_{s f} \equiv \frac{\omega_{\beta \perp}^{2}}{\beta_{b}^{2} c^{2}}, \\
\epsilon^{2} & \equiv 4\left\langle x^{2}+y^{2}\right\rangle\left\langle\frac{p_{x}^{2}+p_{y}^{2}}{\gamma_{b}^{2} m_{b}^{2} \beta_{b}^{2} c^{2}}\right\rangle=\frac{8}{\gamma_{b} m_{b} \beta_{b}^{2} c^{2}} R_{b}^{2}\left\langle T_{\perp b}(r)\right\rangle .
\end{aligned}
$$

Then, making use of Eq. (14), the radial force condition in Eq. (13) can be expressed in the equivalent form

$$
\left(\kappa_{s f}-\frac{K_{b}}{2 R_{b}^{2}}\right) R_{b}=\frac{\epsilon^{2}}{4 R_{b}^{3}},
$$

which is identical to the familiar envelope equation relating the rms beam radius $R_{b}$ to $\kappa_{s f}$, $K_{b}$ and $\epsilon$. For specified values of self-field perveance $\left(K_{b}\right)$, focusing field strength $\left(\kappa_{s f}\right)$, and transverse emittance $(\epsilon)$, Eq. (15) gives a closed expression for the mean-square beam radius,

$$
R_{b}^{2}=\frac{K_{b}}{4 \kappa_{s f}}+\left[\left(\frac{K_{b}}{4 \kappa_{s f}}\right)^{2}+\frac{\epsilon^{2}}{4 \kappa_{s f}}\right]^{1 / 2} .
$$

The radial force balance condition in Eq. (13), or equivalently, Eq. (15), is applicable to the entire class of self-consistent beam equilibria described by Eq. (4), including the special choice of $F_{b}\left(H_{\perp}\right)$ in Eq. (5). Moreover, Eqs. (13) and (15) are applicable over the full range of system parameters ranging from emittance-dominated beams, where

$$
R_{b}^{2} \simeq \frac{2}{\gamma_{b} m_{b} \omega_{\beta \perp}^{2}}\left\langle T_{\perp b}(r)\right\rangle=\frac{\epsilon}{2 \sqrt{\kappa_{s f}}}
$$

for $\left\langle T_{\perp b}(r)\right\rangle \gg N_{b} e_{b}^{2} / 2 \gamma_{b}^{2}$ (or $\epsilon \gg K_{b} / 2 \sqrt{\kappa_{s f}}$ ), to space-charge-dominated beams, where

$$
R_{b}^{2} \simeq \frac{N_{b} e_{b}^{2}}{\gamma_{b}^{3} m_{b} \omega_{\beta \perp}^{2}}=\frac{K_{b}}{2 \kappa_{s f}}
$$

for $N_{b} e_{b}^{2} / 2 \gamma_{b}^{2} \gg\left\langle T_{\perp b}(r)\right\rangle$ (or $K_{b} / 2 \sqrt{\kappa_{s f}} \gg \epsilon$ ).

In analyzing Eqs. (6), (9) and (10) it is important to recognize the powerful constraint on system parameters imposed by the radial force balance condition in Eq. (13) [or Eq. (15)], which relates $R_{b}^{2}, N_{b}$ and $\left\langle T_{\perp b}(r)\right\rangle$. For example, when solving Eq. (9) for $\psi(r)$ for specified value of normalized beam intensity $\hat{s}_{b}=\hat{\omega}_{p b}^{2} / 2 \gamma_{b}^{2} \omega_{\beta \perp}^{2}$, the low-intensity regime $\left(\hat{s}_{b} \ll 1\right)$ corresponds to an emittance dominated beam satisfying Eq. (17). On the other hand, the 
high-intensity limit $\left(\hat{s}_{b} \rightarrow 1\right)$ corresponds to the space-charge-dominated regime satisfying Eq. (18).

In addition to $\hat{s}_{b}$, another convenient measure of self-field intensity is provided by the rms-equivalent tune depression $\nu / \nu_{0}$ defined by

$$
\frac{\nu}{\nu_{0}}=\left(1-\frac{K_{b}}{2 \kappa_{s f} R_{b}^{2}}\right)^{1 / 2}=\left(1-\frac{N_{b} e_{b}^{2}}{\gamma_{b}^{3} m_{b} \omega_{\beta \perp}^{2} R_{b}^{2}}\right)^{1 / 2}
$$

In the low-intensity regime $\left(\hat{s}_{b} \ll 1\right.$ and $\left.K_{b} / 2 \kappa_{s f} R_{b}^{2} \ll 1\right)$ note that Eq. (19) reduces to $\nu / \nu_{0} \simeq 1-K_{b} / 4 \kappa_{s f} R_{b}^{2}$. On the other hand, in the space-charge-dominated limit $\left(\hat{s}_{b} \rightarrow 1\right.$ and $\left.K_{b} / 2 \kappa_{s f} R_{b}^{2} \rightarrow 1\right)$, Eq. (19) reduces to $\nu / \nu_{0} \rightarrow 0$.

\section{EXAMPLES OF EQUILIBRIUM PROFILES}

For specified values of the dimensionless parameters $\hat{s}_{b}=\hat{\omega}_{p b}^{2} / 2 \gamma_{b}^{2} \omega_{\beta \perp}^{2}$ and $E_{b} / \hat{T}_{\perp b}$, Eq. (8) can be solved numerically for the normalized self-field potential $\psi(r) / \hat{T}_{\perp b}$, integrating from $r=0$ where $\psi(r=0)=0=[\partial \psi / \partial r]_{r=0}$. For specified values of $\hat{s}_{b}$ and $E_{b} / \hat{T}_{\perp b}$, note from Poisson's equation (8) that a natural dimensionless radial coordinate is $r / r_{\beta}$ where $r_{\beta}=\left(2 \hat{T}_{\perp b} / \gamma_{b} m_{b} \omega_{\beta \perp}^{2}\right)^{1 / 2}$. Once $\psi(r)$ is determined numerically, the edge radius $r_{b}$ of the beam is obtained from $\psi\left(r=r_{b}\right)=E_{b}$, and the density profile $n_{b}(r)$ and transverse temperature profile $T_{\perp b}(r)$ are determined self-consistently from Eqs. (6) and (10), respectively. Analytical solutions to Eq. (8) are accessible in two limiting cases, which are useful to benchmark the numerical solutions. The first case corresponds to a low-intensity (emittance-dominated) beam with $\hat{s}_{b} \ll 1$ and $\left\langle T_{\perp b}(r)\right\rangle \neq 0$. The second case corresponds to a high-intensity (spacecharge-dominated) beam with $\hat{s}_{b} \rightarrow 1$ and $\left\langle T_{\perp b}(r)\right\rangle \rightarrow 0$.

Low-Intensity Beam Equilibrium $\left(\hat{s}_{b} \ll 1\right)$ : For $\hat{s}_{b} \ll 1$, the space-charge contribution proportional to $\hat{s}_{b}$ in Eq. (8) can be neglected to leading order, which gives $\psi(r) \simeq$ $\left(\gamma_{b} m_{b} \omega_{\beta \perp}^{2} / 2\right) r^{2}$. We determine $r_{b}$ from $\psi\left(r=r_{b-}\right)=E_{b}$, which gives $r_{b}^{2}=2 E_{b} / \gamma_{b} m_{b} \omega_{\beta \perp}^{2}$. Substituting into Eqs. (6) and (10) then gives the density and temperature profiles

$$
n_{b}(r)=\hat{n}_{b} \frac{\left\{\exp \left[-\left(r^{2} / r_{b}^{2}\right)\left(E_{b} / \hat{T}_{\perp b}\right)\right]-\exp \left(-E_{b} / \hat{T}_{\perp b}\right)\right\}}{\left\{1-\exp \left(-E_{b} / \hat{T}_{\perp b}\right)\right\}} \oplus\left(r-r_{b}\right)
$$


and

$T_{\perp b}(r)=\hat{T}_{\perp b} \frac{\left\{\exp \left[\left(-r^{2} / r_{b}^{2}\right)\left(E_{b} / \hat{T}_{\perp b}\right)\right]-\left[1+\left(E_{b} / \hat{T}_{\perp b}\right)\left(1-r^{2} / r_{b}^{2}\right)\right] \exp \left(-E_{b} / \hat{T}_{\perp b}\right)\right\}}{\left\{\exp \left[\left(-r^{2} / r_{b}^{2}\right)\left(E_{b} / \hat{T}_{\perp b}\right)\right]-\exp \left(-E_{b} / \hat{T}_{\perp b}\right)\right\}} \oplus\left(r-r_{b}\right)$.

Illustrative plots of $n_{b}(r) / \hat{n}_{b}$ and $T_{\perp b}(r) / T_{\perp b}(r=0)$ versus $r / r_{b}$ are shown in Fig. 1 for the case where $E_{b} / \hat{T}_{\perp b}=0.1,1,10$. Note from Eqs. (20) and (21) that the beam has a sharp edge at radius $r=r_{b}$, and that the profiles for $n_{b}(r)$ and $T_{\perp b}(r)$ approach zero continuously as $r \rightarrow r_{b}$. Furthermore, in the limit $E_{b} / \hat{T}_{\perp b} \rightarrow \infty$, Eqs. (20) and (21) reduce to the Gaussian density profile $n_{b}(r)=\hat{n}_{b} \exp \left(-\gamma_{b} m_{b} r^{2} \omega_{\beta \perp}^{2} / 2 \hat{T}_{\perp b}\right)$ and uniform temperature profile $T_{\perp b}(r)=\hat{T}_{\perp b}=$ const. On the other hand, for $E_{b} / \hat{T}_{\perp b} \ll 1$, Eqs. (20) and (21) can be approximated by the parabolic profiles $n_{b}(r)=\hat{n}_{b}\left(1-r^{2} / r_{b}^{2}\right)$ and $T_{\perp b}(r)=\left(E_{b} / 2\right)\left(1-r^{2} / r_{b}^{2}\right)$ for $0 \leq r<r_{b}$.

$\underline{\text { Space-Charge-Dominated Beam Equilibrium }\left(\hat{s}_{b} \rightarrow 1,\left\langle T_{\perp b}\right\rangle \rightarrow 0\right)}$ : As a second limiting case that is analytically tractable, we consider Eqs. (5), (6), (8) and (10) in the cold, spacecharge-dominated regime with $\hat{s}_{b}=\hat{\omega}_{p b}^{2} / 2 \gamma_{b}^{2} \omega_{\beta \perp}^{2} \rightarrow 1$, and $\left\langle T_{\perp b}(r)\right\rangle \rightarrow 0$ corresponding to $\hat{T}_{\perp b} \rightarrow 0$ and $\hat{E}_{b} \rightarrow 0$. In this case, the applied focusing force and the (repulsive) spacecharge force exactly cancel in the beam interior, and the solutions to Eqs. (8), (10) and (6) reduce to $\psi(r)=0, T_{\perp b}(r)=0$ and

$$
n_{b}(r)=\hat{n}_{b} \oplus\left(r-r_{b}\right)
$$

for $0 \leq r<r_{b}$. Note from Eq. (21) and Fig. 2 that the beam density profile is uniform with $n_{b}(r)=\hat{n}_{b}=$ const. in the beam interior. Furthermore, for $\hat{s}_{b} \rightarrow 1$, the beam edge radius $r_{b}=\sqrt{2} R_{b}$ is readily determined from $r_{b}^{2}=2 N_{b} e_{b}^{2} / \gamma_{b}^{3} m_{b} \omega_{\beta \perp}^{2}$ [see Eq. (18)], where $N_{b}=\hat{n}_{b} \pi r_{b}^{2}$ is the axial line density.

Comparing Figs. 1 and 2, there is a large variation in the shape of the density profile $n_{b}(r)$ between the low-intensity regime $\left(\hat{s}_{b} \ll 1\right.$ in Fig. 1) and the space-charge-dominated regime $\left(\hat{s}_{b} \rightarrow 1\right.$ in Fig. 2). For general values of $\hat{s}_{b}$ and $E_{b} / \hat{T}_{\perp b}$, Eq. (8) can be solved numerically for $\psi(r)$, and the corresponding self-consistent profiles for $n_{b}(r)$ and $T_{\perp b}(r)$ determined from Eqs. (6) and (8). Typical numerical solutions are illustrated in Figs. 3-6.

Shown in Fig. 3 are plots of the normalized potential $\psi(r) / \hat{T}_{\perp b}$ versus $r / r_{b}$ obtained numerically from Eq. (8) for several values of normalized beam intensity $\hat{s}_{b}=\hat{\omega}_{p b}^{2} / 2 \gamma_{b}^{2} \omega_{\beta \perp}^{2}$ and values of $E_{b} / \hat{T}_{\perp b}$ ranging from 0.1 to 10 . The corresponding values of tune depression $\nu / \nu_{0}$ 

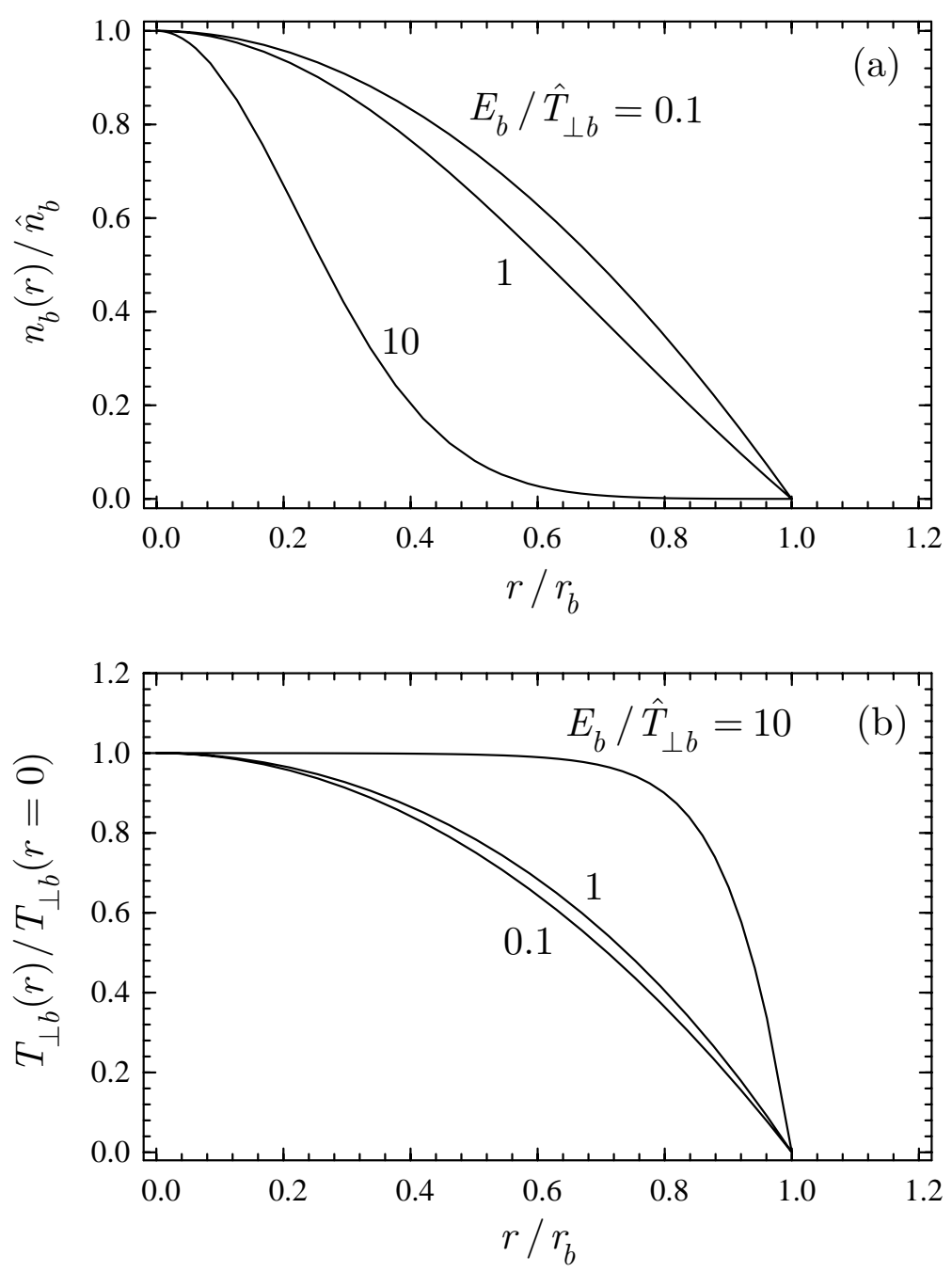

FIG. 1: Plots of normalized (a) density $n_{b}(r) / \hat{n}_{b}$ and (b) temperature $T_{\perp b}(r) / T_{\perp b}(r=0)$ versus $r / r_{b}$ obtained from Eqs. $(20)$ and $(21)$ for $\hat{s}_{b}<<1$, and $E_{b} / \hat{T}_{\perp b}=0.1,1,10$.

[Eq. (19)] are also shown in Fig. 3. Note from Fig. 3 that $\psi(r)$ increases monotonically from $\psi(r=0)=0$ to $\psi\left(r=r_{b}\right)=E_{b}$ at the beam edge. Moreover, comparing Figs. 3(a) and 3(b), $\psi(r)$ is nearly parabolic in the beam interior when $\hat{s}_{b}<<1\left[\hat{s}_{b}=0.1\right.$ in Fig. 3(a)], whereas $\psi(r)$ remains close to zero in the beam interior, and increases rapidly to $\psi\left(r_{b}\right)=E_{b}$ near the beam edge when $\hat{s}_{b} \rightarrow 1\left[\hat{s}_{b}=0.999\right.$ in Fig. 3(d)]. At high space-charge intensity with $\hat{s}_{b} \rightarrow 1$, note from Fig. 3(d) that the effective potential $\psi(r)=\gamma_{b} m_{b} \omega_{\beta \perp}^{2} r^{2} / 2+\left(e_{b} / \gamma_{b}^{2}\right) \phi(r)$ is approximately zero over a broad interior region of the beam, corresponding to a nearcancellation of the applied focusing force and the (repulsive) self-field force. The solutions for $\psi(r) / \hat{T}_{\perp b}$ in Fig. 3 are used to determine the corresponding self-consistent density and 


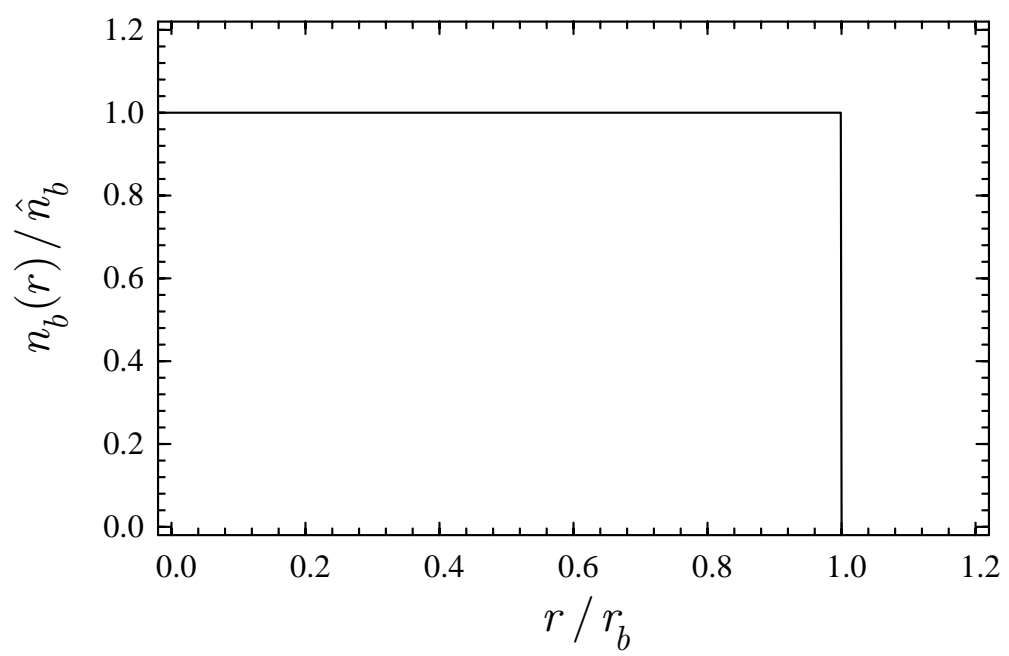

FIG. 2: Plot of normalized density $n_{b}(r) / \hat{n}_{b}$ versus $r / r_{b}$ [Eq. (22)] for space-charge-dominated beam with $\hat{s}_{b} \rightarrow 1$.

temperature profiles from Eqs. (6) and (10). The results are illustrated in Figs. 4 and 5 where the normalized density $n_{b}(r) \pi r_{b}^{2} / N_{b}$ and temperature $T_{\perp b}(r) / \hat{T}_{\perp b}$ are plotted versus $r / r_{b}$ for values of $\hat{s}_{b}$ and $E_{b} / \hat{T}_{\perp b}$ similar to those in Fig. 3. Note from Fig. 4(a) that $n_{b}(r)$ has a diffuse, bell-shaped profile for $\hat{s}_{b}=0.1$, whereas in Fig. $4(\mathrm{~d}) n_{b}(r)$ has an extended flat-top profile in the beam interior for $\hat{s}_{b}=0.999$, as expected. Moreover, from Fig. 5 , the transverse temperature profile $T_{\perp b}(r)$ decreases rather abruptly to zero at the beam edge $\left(r=r_{b}\right)$, although $T_{\perp b}(r)$ tends to be relatively uniform in the beam interior for $E_{b} / \hat{T}_{\perp b}>2$.

Finally, we have made use of Eqs. (6)-(8) and (12) to determine the edge radius $r_{b}$ and rms beam radius $R_{b}$ over a range of system parameters $\hat{s}_{b}$ and $E_{b} / \hat{T}_{\perp b}$. Shown in Fig. 6 are plots of (a) $r_{b} / R_{b}$ versus $\hat{s}_{b}$ and (b) $r_{b} / R_{b}$ versus $\nu / \nu_{0}$ obtained from Eqs. (6)-(8) and Eqs. (12) and (19) for several values of $E_{b} / \hat{T}_{\perp b}$. Note from Fig. 6 that $r_{b} / R_{b}$ is a slowly varying function of $\hat{s}_{b}$ and $\nu / \nu_{0}$, and increases as $E_{b} / \hat{T}_{\perp b}$ is increased.

\section{CONCLUSIONS}

In this paper, we examined properties of the axisymmetric truncated thermal equilibrium distribution defined in Eq. (5). General equilibrium properties were discussed in Secs. 2 and 3 , and expressions for the density profile $n_{b}(r)$ and transverse temperature profile $T_{\perp b}(r)$ were derived in terms of the effective potential $\psi(r)$ [see Eqs. (6) and (10)], where $\psi(r)$ 

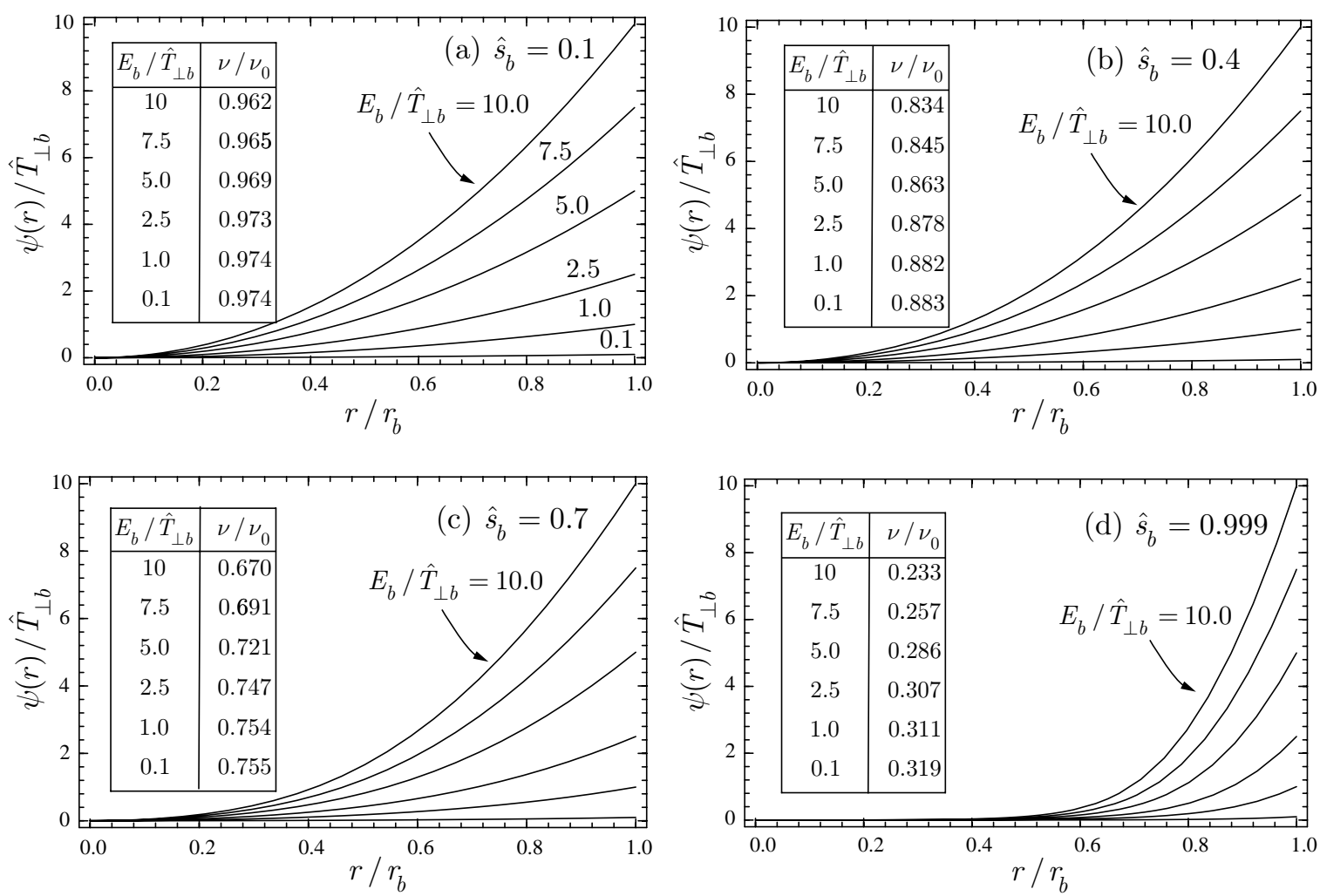

FIG. 3: Plots of normalized potential $\psi(r) / \hat{T}_{\perp b}$ versus $r / r_{b}$ obtained from Eq. (8) for (a) $\hat{s}_{b}=0.1$, (b) $\hat{s}_{b}=0.4$, (c) $\hat{s}_{b}=0.7$ and (d) $\hat{s}_{b}=0.999$, and values of $E_{b} / \hat{T}_{\perp b}$ corresponding to $E_{b} / \hat{T}_{\perp b}=$ $10,7.5,5.0,2.5,1.0,0.1$. The corresponding values of tune depression $\nu / \nu_{0}[\mathrm{Eq} .(19)]$ are also shown in the figure.

solves the nonlinear Poisson equation (8). Detailed numerical solutions to Eqs. (6), (8) and (10) were presented in Sec. 4 for a wide range of dimensionless system parameters corresponding to $s_{b}=\hat{\omega}_{p b}^{2} / 2 \gamma_{b}^{2} \omega_{\beta \perp}^{2}$ and $E_{b} / \hat{T}_{\perp b}$. Several properties of the equilibrium profiles are noteworthy. For example, the beam has a sharp outer edge radius $r_{b}$ with $n_{b}\left(r \geq r_{b}\right)=0$, where $r_{b}$ depends on the value of $E_{b} / \hat{T}_{\perp b}$. In addition, unlike the choice of a semi-Gaussian distribution, $F_{b}^{S G}=A \exp \left(-p_{\perp}^{2} / 2 \gamma_{b} m_{b} \hat{T}_{\perp b}\right) \oplus\left(r-r_{b}\right)$, the truncated thermal equilibrium distribution in Eq. (5) depends on $\left(r, \mathbf{p}_{\perp}\right)$ only through the single-particle of the motion $H_{\perp}$ and is therefore a true steady-state solution $(\partial / \partial t=0)$ of the nonlinear Vlasov-Maxwell equations. It is anticipated that the choice of distribution function in Eq. (5) will be useful in implementing particle-in-cell simulations of intense beams without large initial transient evolutions, and incorporating the important feature of a beam with sharp outer edge radius. 

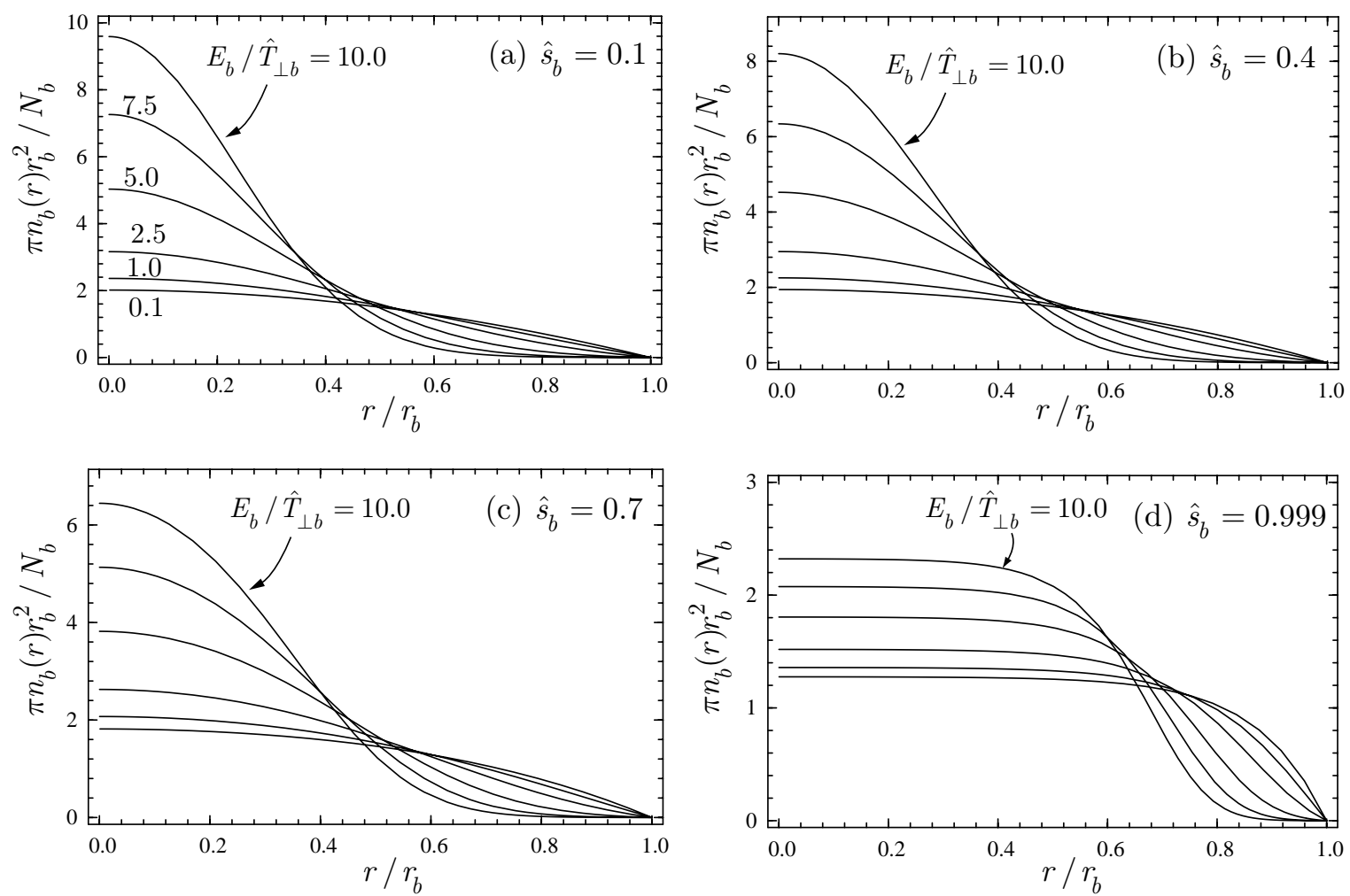

FIG. 4: Plots of normalized density profile $n_{b}(r) \pi r_{b}^{2} / N_{b}$ versus $r / r_{b}$ obtained from Eqs. (6) and (8) for $(\mathrm{a}) \hat{s}_{b}=0.1$, (b) $\hat{s}_{b}=0.4$, (c) $\hat{s}_{b}=0.7$ and (d) $\hat{s}_{b}=0.999$, and several values of $E_{b} / \hat{T}_{\perp b}$ and $\nu / \nu_{0}$ identical to those in Fig. 3.

Finally, it should be pointed out that the present analysis also applies to an intense rotating beam propagating through a uniform solenoidal magnetic field $B_{0} \hat{\mathbf{e}}_{z}$ in circumstances where the beam particles have zero average canonical angular momentum $<P_{\theta}>=r(<$ $\left.p_{\theta}>+e_{b} B_{0} r / 2 c\right)=0$. In this case, we make the replacement $\omega_{\beta \perp} \rightarrow \omega_{c b} / 2$, and all variables are interpreted as being measured in a frame of reference rotating with the Larmor frequency $\omega_{c b} / 2=-e_{b} B_{0} / 2 \gamma_{b} m_{b} c$ relative to the laboratory frame. 

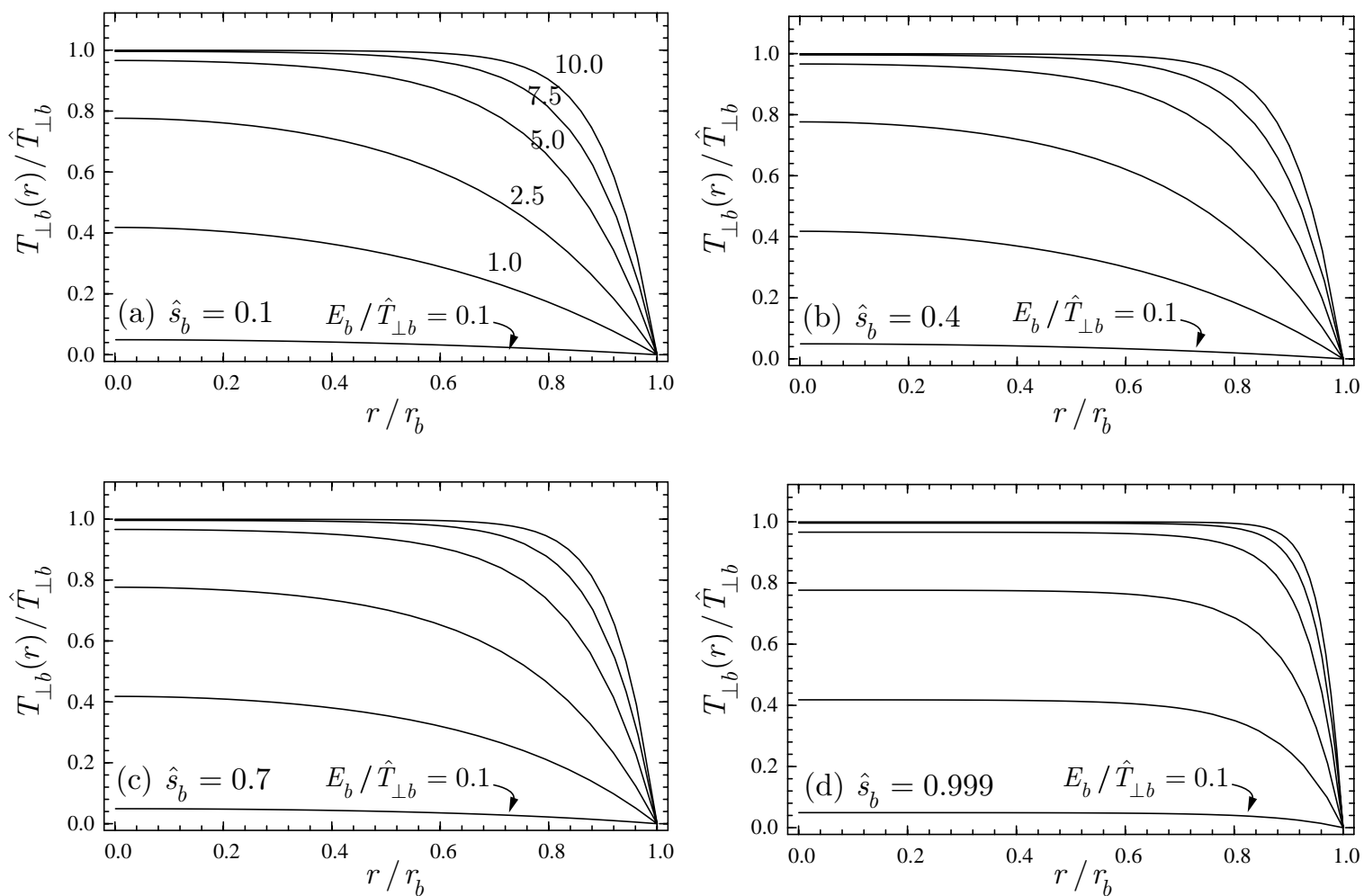

FIG. 5: Plots of normalized temperature profile $T_{\perp b}(r) / \hat{T}_{\perp b}$ versus $r / r_{b}$ obtained from Eqs. (8) and (10) for (a) $\hat{s}_{b}=0.1$, (b) $\hat{s}_{b}=0.4$, (c) $\hat{s}_{b}=0.7$ and (d) $\hat{s}_{b}=0.999$, and several values of $E_{b} / \hat{T}_{\perp b}$ and $\nu / \nu_{0}$ identical to those in Fig. 3.

\section{Acknowledgments}

This research was supported by the U.S. Department of Energy under contract AC0276CH03073. .

[1] R. C. Davidson and H. Qin, Physics of Intense Charged Particle Beams in High Energy Accelerators (World Scientific, 2001), and references therein.

[2] R. C. Davidson, Physics of Nonneutral Plasmas (World Scientific, 2001), and references therein.

[3] A. W. Chao, Physics of Collective Beam Instabilities in High Energy Accelerators (Wiley, New York, 1993).

[4] M. Reiser, Theory and Design of Collective Particle Beams (Wiley, New York, 1994). 

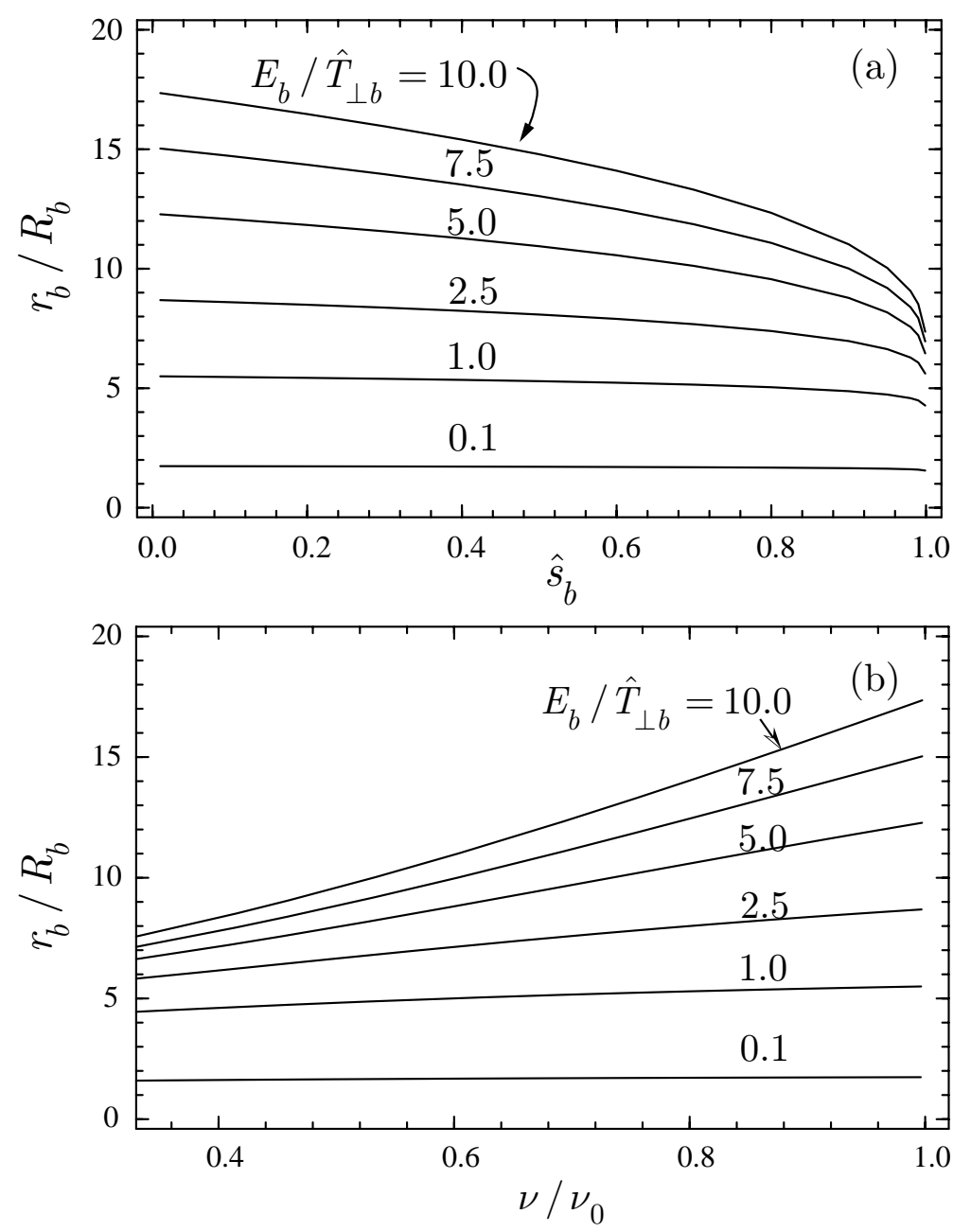

FIG. 6: Plots of normalized edge radius (a) $r_{b} / R_{b}$ versus $\hat{s}_{b}$ and (b) $r_{b} / R_{b}$ versus $\nu / \nu_{0}$ obtained from Eqs. (6)-(8) and Eqs. (12) and (19) for several values of $E_{b} / \hat{T}_{\perp b}$ ranging from 0.1 to 10 .

[5] A. Friedman, D. P. Grote and I. Haber, Phys. Fluids B4, 2203 (1992).

[6] S. M. Lund, J. J. Barnard, G. D. Craig, A. Friedman, D. P. Grote, T. S. Sangster, W. M. Sharp, S. Eylon, T. T. Fessenden, E. Henestroza, S. Yu and I. Jaber, Nuclear Instruments and Methods in Physics Research A415, 345 (1998).

[7] A. Friedman, J. J. Barnard, D. P. Grote and I. Haber, Nuclear Instruments and Methods in Physics Research A415, 455 (1998).

[8] I. Haber, A. Friedman, D. P. Grote, S. M. Lund and R. A. Kishek, Phys. Plasmas 6, 2254 (1999).

[9] E. Sonnendrucker, J. J. Barnard, A. Friedman, D. P. Grote and S. M. Lund, Nuclear Instru- 
ments and Methods in Physics Research A464, 470 (2001); R. A. Kishek, P. G. O'Shea and M. Reiser, Phys. Rev. Lett. 85, 4514 (2000).

[10] See, for example, Chapters 5 and 7 of Ref. 1.

[11] R. C. Davidson and N. A. Krall, Phys. Fluids 13, 1543 (1970).

[12] I. Hofmann and J. Struckmeier, Part. Accel. 21, 69 (1987).

[13] J. Struckmeier and I. Hofmann, Part. Accel. 39, 219 (1992).

[14] R. C. Davidson and S. M. Lund, in Advances in Plasma Physics, edited by N. J. Fisch, AIP Conf. Proc. 314 (AIP, New York, 1994), p. 1.

[15] N. Brown and M. Reiser, Phys. Plasmas 2, 965 (1995).

[16] R. C. Davidson and C. Chen, Part. Accel. 59, 175 (1998).

[17] R. C. Davidson, Phys. Rev. Lett. 81, 991 (1998).

[18] R. C. Davidson and H. Qin, Phys. Rev. ST Accel. Beams 2, 114401 (1999).

[19] D. H. E. Dubin and T. M. O’Neil, Rev. Mod. Phys. 71, 20 (1999).

[20] R. C. Davidson, W. W. Lee and P. H. Stoltz, Phys. Plasmas 5, 279 (1998).

[21] See, for example, Chapter 6 of Ref. 1. 


\section{External Distribution}

Plasma Research Laboratory, Australian National University, Australia

Professor I.R. Jones, Flinders University, Australia

Professor João Canalle, Instituto de Fisica DEQ/IF - UERJ, Brazil

Mr. Gerson O. Ludwig, Instituto Nacional de Pesquisas, Brazil

Dr. P.H. Sakanaka, Instituto Fisica, Brazil

The Librarian, Culham Laboratory, England

Mrs. S.A. Hutchinson, JET Library, England

Professor M.N. Bussac, Ecole Polytechnique, France

Librarian, Max-Planck-Institut für Plasmaphysik, Germany

Jolan Moldvai, Reports Library, MTA KFKI-ATKI, Hungary

Dr. P. Kaw, Institute for Plasma Research, India

Ms. P.J. Pathak, Librarian, Insitute for Plasma Research, India

Ms. Clelia De Palo, Associazione EURATOM-ENEA, Italy

Dr. G. Grosso, Instituto di Fisica del Plasma, Italy

Librarian, Naka Fusion Research Establishment, JAERI, Japan

Library, Plasma Physics Laboratory, Kyoto University, Japan

Research Information Center, National Institute for Fusion Science, Japan

Dr. O. Mitarai, Kyushu Tokai University, Japan

Dr. Jiangang Li, Institute of Plasma Physics, Chinese Academy of Sciences, People's Republic of China

Professor Yuping Huo, School of Physical Science and Technology, People's Republic of China

Library, Academia Sinica, Institute of Plasma Physics, People's Republic of China

Librarian, Institute of Physics, Chinese Academy of Sciences, People's Republic of China

Dr. S. Mirnov, TRINITI, Troitsk, Russian Federation, Russia

Dr. V.S. Strelkov, Kurchatov Institute, Russian Federation, Russia

Professor Peter Lukac, Katedra Fyziky Plazmy MFF UK, Mlynska dolina F-2, Komenskeho Univerzita, SK-842 15 Bratislava, Slovakia

Dr. G.S. Lee, Korea Basic Science Institute, South Korea

Institute for Plasma Research, University of Maryland, USA

Librarian, Fusion Energy Division, Oak Ridge National Laboratory, USA

Librarian, Institute of Fusion Studies, University of Texas, USA

Librarian, Magnetic Fusion Program, Lawrence Livermore National Laboratory, USA

Library, General Atomics, USA

Plasma Physics Group, Fusion Energy Research Program, University of California at San Diego, USA

Plasma Physics Library, Columbia University, USA

Alkesh Punjabi, Center for Fusion Research and Training, Hampton University, USA

Dr. W.M. Stacey, Fusion Research Center, Georgia Institute of Technology, USA

Dr. John Willis, U.S. Department of Energy, Office of Fusion Energy Sciences, USA

Mr. Paul H. Wright, Indianapolis, Indiana, USA 
The Princeton Plasma Physics Laboratory is operated by Princeton University under contract with the U.S. Department of Energy.

\author{
Information Services \\ Princeton Plasma Physics Laboratory \\ P.O. Box 451 \\ Princeton, NJ 08543
}

Phone: 609-243-2750

Fax: 609-243-2751

e-mail: pppl_info@pppl.gov

Internet Address: http://www.pppl.gov 